Jan Camenisch

Doğan Kesdoğan (Eds.)

\title{
Open Problems in Network Security
}

IFIP WG 11.4 International Workshop, iNetSec 2017

Rome, Italy, May 2017

Proceedings 
Volume Editors

Jan Camenisch

IBM Research - Zurich

Säumerstr. 4

8803 Rüschlikon, Switzerland

E-mail: jca@zurich.ibm.com

Doğan Kesdoğan

Department of Business Information Systems 4

University of Regensburg

93040 Regensburg, Germany

E-mail: kesdogan@ur.de 


\section{Preface}

In the past decade, computer networks have fundamentally influenced the ways in which information is exchanged and handled. Pervasive electronic devices make our everyday life easier, e.g., by helping us to connect with other people while being mobile, to use services available online, or to pay and use tolls and tickets. Many of the components employed routinely manage and distribute large amounts of data for different purposes. As these processes involve sensitive information, protecting information and the network with suitable security measures is more important than ever.

The objective of this workshop is to bring together researchers in the field of network security to discuss the open problems and future research directions. 


\section{Open Problems in Network Security \\ IFIP WG 11.4 International Workshop, iNetSec 2017 \\ Rome, Italy, May 2017}

\section{Program Chair}

Doğan Kesdoğan

University of Regensburg, Germany

\section{Organising Chair}

Vinh Pham

University of Regensburg, Germany

\section{Program Committee}

Muhammad Ajmal

Roberto Di Pietro

Hannes Federrath

Danilo Gligoroski

Doğan Kesdoğan

Albert Levi

Giovanni Livraga

Javier Lopez

Vinh Pham

Kai Rannenberg

Siraj A. Shaikh

Newcastle University, UK

Nokia Bell Labs, France

University of Hamburg, Germany

NTNU, Norway

University of Regensburg, Germany

Sabanci University, Turkey

University of Milan, Italy

University of Malaga, Spain

University of Regensburg, Germany

Goethe University Frankfurt, Germany

Coventry University, UK 


\section{Table of Contents}

Trust-Aware Security for Disruption-Tolerant Networks . . . . . . . . 1 Vladimir Oleshchuk

Post Disaster Resilient Networks: Design Guidelines for Rescue

Operations ..................... 10

Tooska Dargahi, Sadaf Momeni, and Hosein Shafiei

Blockchain-based Public Key Infrastructure for Inter-Domain Secure

Routing ...................... 20 20

Alfonso de la Rocha Gómez-Arevalillo and Panos Papadimitratos

The Challenge of Private Identification . . . . . . . . . . . . . . . . . . 39

Stig F. Mjølsnes and Ruxandra F. Olimid

Understanding Trustworthy Service Level Agreements: Open

Problems and Existing Solutions . . . . . . . . . . . . . 54

Yudhistira Nugraha and Andrew Martin 